\section{Monitoring of irradiated food products marketed in Italy and evaluation of electron spin resonance signal sensitivity of experimentally irradiated fish scales}

Raffaele Marrone ${ }^{1}$ Leonardo Carosielti, Michele Mangiacotti, ${ }^{3}$

Eugenio Chiaravalle, ${ }^{3}$ Giorgio Smaldone, ${ }^{1}$ Aniello Anastasio ${ }^{1}$

'Dipartimento di Medicina Veterinaria e Produzioni Animali, Università degli Studi di Napoli Federico II; ${ }^{2}$ Centro di Referenza Nazionale per la Ricerca della Radioattività nel Settore Zootecnico Veterinario, Istituto Zooprofilattico Sperimentale della Puglia e Basilicata, Foggia; ${ }^{3}$ Servizio Veterinario, Azienda Sanitaria Locale di Foggia, Italy

\section{Abstract}

Many countries, in order to authorise the use of food irradiation, claim the availability of methods to detect the occurred treatment in addition to the respect of safe use of this technology. Among physical methods, the electron spin resonance (ESR) measuring the number of free radicals that are formed during irradiation can be applied only to those foods with cellulose, a crystalline or bone structure, in which free radicals have a shelf life greater than irradiated product. The aim of this study was to highlight an irradiation treatment in European and extra-European foods marketed in Southern Italy by the means of ESR technique. Furthermore, in order to optimise the preparation procedures the efficacy of the above mentioned method in fish scales experimentally irradiated has been evaluated. From February to September 2012, a total number of 83 samples of food products of animal and plant origin were taken at the border inspection post and at retail market and finally analysed. At the same time, the scales of grouper and barracuda have been experimentally irradiated at $0.5 \mathrm{kGy}$ and were subsequently analysed using ESR. Results showed 5 frog legs out of 83 samples positive for treatment and confirm the applicability of ESR also for fish scales.

\section{Introduzione}

Attualmente sono disponibili numerosi sistemi di conservazione degli alimenti e la scelta del più idoneo dipende dal tipo di alimento e da fattori igienico-sanitari, ambientali, economici e anche psicologici. Accanto ai metodi tradizionali ha destato un crescente interesse l'utilizzo delle radiazioni ionizzanti che esplicano la loro azione conservativa in diversi modi interferendo in maniera marginale sull'attività enzimatica degli alimenti (Codex Alimentarius Commission; FA0, 1984). Queste hanno la potenzialità di diminuire 0 eliminare la contaminazione microbica prevenendo il deterioramento. Dall'inizio della sua applicazione ad oggi il trattamento degli alimenti con radiazioni ionizzanti ha coinvolto un numero sempre maggiore di Paesi e sempre più diverse tipologie alimentari. Nonostante il Comitato Scientifico dell'Alimentazione Umana, basandosi sui lavori della Food and Agriculture Organization of the United Nations/International Atomic Energy Agency/World Health Organization (FAO/LAEA/WHO), abbia affermato che l'irraggiamento delle derrate alimentari fino alla dose massima di $10 \mathrm{kGy}$ non presenti problemi di natura tossicologica, nutrizionale e microbiologica (WHO, 1999), il trattamento con radiazioni ionizzanti ha provocato e continua a suscitare ancora oggi reazioni controverse. Molti Paesi, oltre a richiedere le necessarie valutazioni sulla sicurezza d'uso degli alimenti irradiati, hanno posto come prerequisito per l'autorizzazione all'irraggiamento, la disponibilità di metodi atti a rilevare l'applicazione del trattamento stesso (Farkas, 1998; Stefanova et al., 2010). Tali metodi devono offrire la possibilità di verificare inequivocabilmente se un alimento sia stato trattato o meno con radiazioni ionizzanti e devono poter essere applicati sull'alimento in fase di commercializzazione. L'identifi-cazione degli alimenti irradiati deve permettere quindi una verifica a posteriori che si distingue nettamente dalle verifiche effettuate direttamente negli impianti di irraggiamento. Tutto ciò ha portato ad un sempre maggiore interessamento per la ricerca di metodiche analitiche che rispondessero adeguatamente a tali richieste. I metodi validati e standardizzati dal Comitato Europeo di Normazione (CEN) possono essere suddivisi in base alle modificazioni radio-indotte sull'alimento in metodi chimici, fisici e biologici (Delincée, 2002; Boniglia et al., 2004). Tra i metodi fisici, la tecnica della risonanza di spin elettronico (electron spin resonance; ESR) (Ikeya, 1993; Eaton et al., 1998), misurando la concentrazione dei radicali liberi che si formano in seguito all'irraggiamento, può essere applicata solo a quegli alimenti contenenti zuccheri cristallini (EN 13708; Commissione Europea, 2001), cellulosa (EN 1787; Commissione Europea, 2000) o matrice ossea (EN 1786; Commissione Europea, 1996) in cui i radicali liberi hanno un tempo
Correspondence: Raffaele Marrone, Dipartimento di Medicina Veterinaria e Produzioni Animali, Università degli Studi di Napoli Federico II, via Delpino 1, 80137 Napoli, Italy.

Tel. +39.081.2536464 - Fax: +39.081.458683.

E-mail: raffaele.marrone@unina.it

Key words: Food irradiation, Food safety, Electron spin resonance, Fish scales.

Received for publication: 11 May 2013. Accepted for publication: 6 October 2013.

This work is licensed under a Creative Commons Attribution 3.0 License (by-nc 3.0).

(C) Copyright R. Marrone et al., 2014

Licensee PAGEPress, Italy

Italian Journal of Food Safety 2014; 3:1602

doi:10.4081/ijfs.2014.1602

di vita superiore alla shelf life del prodotto trattato. Alla luce di quanto esposto, obiettivo di questo lavoro è stato quello di evidenziare un eventuale trattamento di irraggiamento in alimenti commercializzati sul territorio italiano, in particolare nella provincia di Napoli, di provenienza europea ed extra europea. Parallelamente, al fine di estendere il campo di applicazione del metodo (EN 1786; Commissione Europea, 1996) e ridurre i tempi di analisi, è stata valutata l'applicabilità della tecnica ESR a squame di pesci provenienti da cernia e barracuda, suscettibili di essere sottoposti a trattamento.

\section{Materiali e Metodi}

\section{Sperimentazione A:}

campionamento alimenti

commercializzati sul territorio

\section{italiano}

Da febbraio a settembre 2012 sono stati prelevati presso il posto di Ispezione ispezione frontaliera (PIF) di Napoli, presso esercizi commerciali presenti sul territorio della provincia di Napoli e sul territorio nazionale, 83 campioni di prodotti di origine animale $\mathrm{e}$ vegetale suddivisi per tipologia come riportato in Tabella 1. I campioni di origine animale sono stati immediatamente congelati e stoccati a temperatura di $-80^{\circ} \mathrm{C}$. Successivamente sono stati trasferiti presso il Centro di Referenza Nazionale per la Ricerca della Radioattività nel settore ZootecnicoVeterinario dell'Istituto Zooprofilattico Sperimentale della Puglia e della Basilicata, sede di Foggia ed analizzati con i metodi ufficiali basati sulla tecnica ESR, mediante uno spettrometro BRUKER, modello EMX-113. 
Tabella 1. Descrizione e provenienza degli 83 campioni con relativi esiti analitici.

\begin{tabular}{|c|c|c|c|c|}
\hline Matrice & Categoria & Provenienza & Confezionamento & Analisi di conferma \\
\hline Zucchero marrone & Altro & Cina & Prodotto sfuso & Non irraggiato \\
\hline Basilico & Erbe e spezie & Nord Africa & Prodotto sfuso & Non irraggiato \\
\hline Condimento barbecue & Erbe e spezie & Italia & Prodotto sfuso & Non irraggiato \\
\hline Pepe nero & Erbe e spezie & Vietnam & Prodotto sfuso & Non irraggiato \\
\hline Peperoncino & Erbe e spezie & India & Prodotto sfuso & Non irraggiato \\
\hline Arachidi & Frutta & USA & Prodotto sfuso & Non irraggiato \\
\hline Arachidi & Frutta & Italia & Prodotto sfuso & Non irraggiato \\
\hline Arachidi & Frutta & Italia & Prodotto sfuso & Non irraggiato \\
\hline Arachidi tostate & Frutta & Italia & Prodotto sfuso & Non irraggiato \\
\hline Fragole & Frutta & Italia & Prodotto sfuso & Non irraggiato \\
\hline Fragole & Frutta & Italia & Prodotto sfuso & Non irraggiato \\
\hline Litchi & Frutta & Cina & Prodotto sfuso & Non irraggiato \\
\hline Mandorle & Frutta & Italia & Prodotto sfuso & Non irraggiato \\
\hline Nocciole & Frutta & Italia & Prodotto sfuso & Non irraggiato \\
\hline Noci & Frutta & USA & Prodotto sfuso & Non irraggiato \\
\hline Noci & Frutta & Italia & Prodotto sfuso & Non irraggiato \\
\hline Noci brasiliane & Frutta & Brasile & Prodotto sfuso & Non irraggiato \\
\hline Noci della California & Frutta & USA & Prodotto sfuso & Non irraggiato \\
\hline Noci moscate intere & Frutta & Paesi fascia tropicale & Prodotto sfuso & Non irraggiato \\
\hline Pistacchi & Frutta & California & Prodotto sfuso & Non irraggiato \\
\hline Pistacchi tostati & Frutta & Iran & Prodotto sfuso & Non irraggiato \\
\hline Pistacchi tostati & Frutta & Iran & Prodotto sfuso & Non irraggiato \\
\hline Agnello & Prodotti carnei & Nuova Zelanda & Sottovuoto & Non irraggiato \\
\hline Alette di pollo & Prodotti carnei & Francia & Sottovuoto & Non irraggiato \\
\hline Anatra & Prodotti carnei & Ungheria & Sottovuoto & Non irraggiato \\
\hline Bovino & Prodotti carnei & Regno Unito & Sottovuoto & Non irraggiato \\
\hline Bovino & Prodotti carnei & Francia & Sottovuoto & Non irraggiato \\
\hline Bovino & Prodotti carnei & Italia & Sottovuoto & Non irraggiato \\
\hline Braciola di vitello & Prodotti carnei & Francia & Sottovuoto & Non irraggiato \\
\hline Cosce di rana & Prodotti carnei & Albania & Sottovuoto & Non irraggiato \\
\hline Cosce di rana & Prodotti carnei & Vietnam & Sottovuoto & Irraggiato \\
\hline Cosce di rana & Prodotti carnei & Turchia & Sottovuoto & Non irraggiato \\
\hline Cosce di rana & Prodotti carnei & Vietnam & Sottovuoto & Irraggiato \\
\hline Cosce di rana & Prodotti carnei & Vietnam & Sottovuoto & Non irraggiato \\
\hline Cosce di rana & Prodotti carnei & Vietnam & Sottovuoto & Irraggiato \\
\hline Cosce di rana & Prodotti carnei & Vietnam & Sottovuoto & Non irraggiato \\
\hline Cosce di rana & Prodotti carnei & Vietnam & Sottovuoto & Non irraggiato \\
\hline Cosce di rana & Prodotti carnei & Vietnam & Sottovuoto & Non irraggiato \\
\hline Cosce di rana & Prodotti carnei & Vietnam & Prodotto sfuso & Irraggiato \\
\hline Cosce di rana & Prodotti carnei & Turchia & Prodotto sfuso & Non irraggiato \\
\hline Cosce di rana & Prodotti carnei & Vietnam & Prodotto sfuso & Irraggiato \\
\hline Muscolo bovino & Prodotti carnei & Polonia & Prodotto sfuso & Non irraggiato \\
\hline Muscolo di suino & Prodotti carnei & Olanda & Prodotto sfuso & Non irraggiato \\
\hline Muscolo ovino & Prodotti carnei & Nuova Zelanda & Prodotto sfuso & Non irraggiato \\
\hline Ovino & Prodotti carnei & Nuova Zelanda & Prodotto sfuso & Non irraggiato \\
\hline Pollo & Prodotti carnei & Germania & Prodotto sfuso & Non irraggiato \\
\hline Pollo & Prodotti carnei & Spagna & Sottovuoto & Non irraggiato \\
\hline Pollo & Prodotti carnei & Francia & Sottovuoto & Non irraggiato \\
\hline Pollo & Prodotti carnei & Italia & Sottovuoto & Non irraggiato \\
\hline Pollo & Prodotti carnei & Italia & Sottovuoto & Non irraggiato \\
\hline Suino & Prodotti carnei & Italia & Sottovuoto & Non irraggiato \\
\hline
\end{tabular}


Tabella 1. Continua dalla pagina precedente.

\begin{tabular}{|c|c|c|c|c|}
\hline Matrice & Categoria & Provenienza & Confezionamento & Analisi di conferma \\
\hline Cosce di rana & Prodotti carnei & Vietnam & Sottovuoto & Non irraggiato \\
\hline Acciuga & Prodotti ittici & Cina & Prodotto sfuso & Non irraggiato \\
\hline Acciuga giapponese & Prodotti ittici & Giappone & Prodotto sfuso & Non irraggiato \\
\hline Anguilla & Prodotti ittici & Nuova Zelanda & Prodotto sfuso & Non irraggiato \\
\hline Barracuda & Prodotti ittici & Corea & Prodotto sfuso & Non irraggiato \\
\hline Cernia & Prodotti ittici & Corea & Prodotto sfuso & Non irraggiato \\
\hline Code gamberi & Prodotti ittici & Tailandia & Sottovuoto & Non irraggiato \\
\hline Corvina & Prodotti ittici & Cina & Prodotto sfuso & Non irraggiato \\
\hline Cozze & Prodotti ittici & Cile & Prodotto sfuso & Non irraggiato \\
\hline Gamberi black tiger & Prodotti ittici & Cile & Prodotto sfuso & Non irraggiato \\
\hline Latterini & Prodotti ittici & Turchia & Sottovuoto & Non irraggiato \\
\hline Pernice & Prodotti ittici & Gran Bretagna & Prodotto sfuso & Non irraggiato \\
\hline Pesce gatto affumicato & Prodotti ittici & Gambia & Sottovuoto & Non irraggiato \\
\hline Pesce imperatore & Prodotti ittici & Corea del sud & Sottovuoto & Non irraggiato \\
\hline Pesce pappagallo & Prodotti ittici & Bangladesh & Sottovuoto & Non irraggiato \\
\hline Pesce persico & Prodotti ittici & Bangladesh & Sottovuoto & Non irraggiato \\
\hline Puntine di maiale & Prodotti ittici & Olanda & Sottovuoto & Non irraggiato \\
\hline Rana pescatrice & Prodotti ittici & Cina & Prodotto sfuso & Non irraggiato \\
\hline Rana pescatrice & Prodotti ittici & Cina & Prodotto sfuso & Non irraggiato \\
\hline Rana pescatrice & Prodotti ittici & Olanda & Prodotto sfuso & Non irraggiato \\
\hline Ricciola & Prodotti ittici & Oman & Prodotto sfuso & Non irraggiato \\
\hline Rombo & Prodotti ittici & Olanda & Prodotto sfuso & Non irraggiato \\
\hline Salmone & Prodotti ittici & Norvegia & Prodotto sfuso & Non irraggiato \\
\hline Seppie & Prodotti ittici & Indonesia & Prodotto sfuso & Non irraggiato \\
\hline Seppie & Prodotti ittici & Vietnam & Prodotto sfuso & Non irraggiato \\
\hline Tilapia & Prodotti ittici & Tailandia & Prodotto sfuso & Non irraggiato \\
\hline Triglie & Prodotti ittici & Mauritania & Prodotto sfuso & Non irraggiato \\
\hline Pesce corvina & Prodotti ittici & Cina & Prodotto sfuso & Non irraggiato \\
\hline Frumento & Vegetali & Italia & Prodotto sfuso & Non irraggiato \\
\hline Frumento & Vegetali & Canada & Prodotto sfuso & Non irraggiato \\
\hline Frumento & Vegetali & Canada & Prodotto sfuso & Non irraggiato \\
\hline Frumento & Vegetali & Canada & Prodotto sfuso & Non irraggiato \\
\hline
\end{tabular}

\section{Sperimentazione B:}

\section{applicazione tecnica ESR in pesci sperimentalmente irradiati}

L'applicazione della tecnica ESR su alimenti contenenti tessuto cristallino (ossa, lische di pesce) prevede una fase preparativa estremamente indaginosa, al fine di ottenere la frazione minerale (idrossiapatite) il più possibile priva di parti organiche. Pertanto, con il duplice scopo di ridurre i tempi di analisi ed estendere il campo di applicazione del metodo (EN 1786; Commissione Europea, 1996) a matrici ittiche non ancora validate, per questo disegno sperimentale sono state irradiate 10 squame di pesci (da una cernia e da un barracuda), risultati negativi alla precedente analisi. Tali matrici, sperimentalmente irraggiate alla dose di 0,5 kGy, utilizzando un irraggiatore a raggi a bassa energia (d.d.p. $<150 \mathrm{kV}$ ) RAD SOURCE Inc.
Mod. RS 2400, sono state successivamente analizzate secondo il metodo descritto nella sperimentazione A.

\section{Risultati}

\section{Sperimentazione A}

Su 83 campioni analizzati, 5 campioni di cosce di rana provenienti dal Vietnam sono risultati positivi. Tutti i 5 campioni sono risultati non conformi in quanto non riportavano in etichetta il trattamento con radiazioni ionizzanti.

\section{Sperimentazione B}

Nelle Figure 1 e 2 sono riportati, a titolo di esempio, gli spettri ESR delle squame di barra- cuda, rispettivamente non irradiate e irradiate. Da esse si nota nel campione di controllo (non irradiato), oltre al segnale del marker, sempre presente nella parte destra degli spettri, un segnale simmetrico (Figura 1), mentre in quello trattato si nota un debole segnale attribuibile all'idrossiapatite (Figura 2), sovrapposto al segnale nativo tipico degli alimenti non trattati.

\section{Discussione}

I risultati delle nostre indagini mostrano la presenza di alimenti irradiati e non correttamente etichettati sul nostro territorio a conferma di quanto riportato nelle recenti allerte comunitarie (Commissione Europea, 2012) ed 
evidenziano la necessità di indirizzare i campionamenti sulle tipologie di prodotti già risultati positivi in precedenti indagini ufficiali (Mangiacotti et al., 2013) o provenienti da Paesi, come il Vietnam, in cui sono attivi numerosi impianti di irraggiamento. Il monitoraggio effettuato su prodotti alimentari pre- senti in fase di commercializzazione sul territorio italiano tramite i metodi ufficiali, conferma l'affidabilità della tecnica ESR nel controllo ufficiale degli alimenti irradiati non correttamente etichettati o non autorizzati nel territorio dell'Unione europea. Inoltre, la sperimentazione condotta su squame di pesci sperimen-

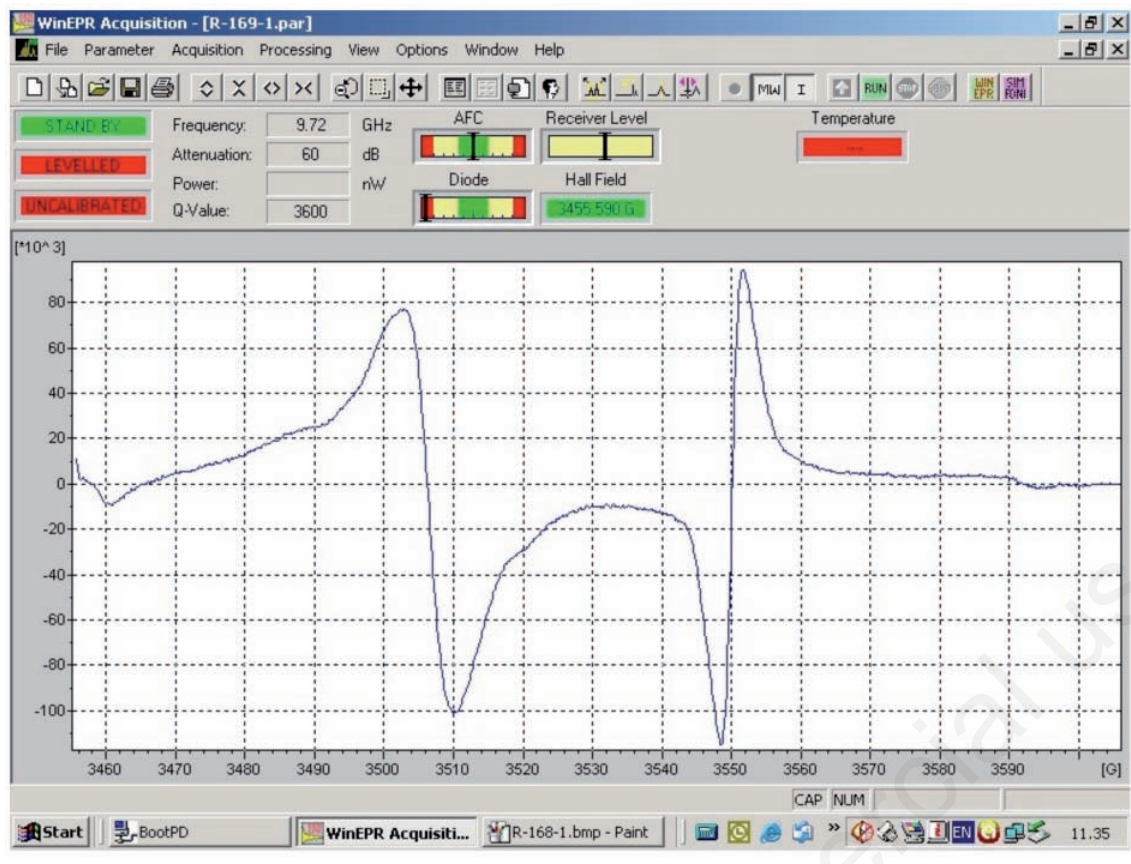

Figura 1. Spettro relativo alle squame di barracuda. talmente trattate, pur nei limiti di una ridotta casistica, costituisce una solida base di partenza per estendere il campo di applicazione di metodi già normati ad un settore, quello ittico, in cui il ricorso al trattamento radiante è sempre più frequente. Importante dal punto di vista pratico è anche la riduzione dei tempi di analisi del metodo adottato, in quanto l'applicazione del metodo standard (EN 1786; Commissione Europea, 1996) a matrici complesse quali i pesci richiede normalmente operazioni lunghe per l'estrazione e pulizia della lisca prima di sottoporla alla determinazione analitica. In accordo con i recenti pareri espressi dai panel di esperti della European Food Safety Authority (2011), che hanno esaminato l'efficacia e la sicurezza microbiologica del processo ed i possibili rischi connessi alla formazione di numerose sostanze chimiche prodotte in seguito al trattamento di irradiazione degli alimenti, con conclusioni rassicuranti sull'utilizzo di alimenti irradiati nei limiti stabiliti dall'attuale normativa comunitaria vigente (Direttive 1999/2/CE e1999/3/CE; Commissione Europea, 1999a, 199b) e recepita nell'ordinamento nazionale (D. Lgs.vo $\mathrm{n}^{\circ}$ 94/2001; Repubblica Italiana, 2001), la pratica dell'irradiazione, benché efficace, deve considerarsi soltanto uno dei numerosi processi che possono ridurre la presenza di patogeni negli alimenti.

\section{Conclusioni}

In conclusione, l'irraggiamento l'irraggiamento degli alimenti od ingredienti alimentari deve essere considerato parte di un programma integrato di gestione della sicurezza alimentare, oltre che oggetto di analisi e riduzione del rischio, volto a proteggere i consumatori, che comprende buone prassi agricole, produttive e igieniche e applicazione del sistema HACCP. Inoltre, al fine di garantire la libertà di scelta del consumatore ed il rispetto della lista positiva di alimenti ammessi al trattamento negli impianti ed alle dosi autorizzate, devono essere rafforzati i controlli ufficiali nel campo della l'irraggiamento food irradiationalimentare.

\section{Bibliografia}

Boniglia C, Onori S, Sapora 0, 2004. Trattamento degli alimenti con radiazioni ionizzanti. Istituto Superiore di Sanità, Roma, Italia.

Commissione Europea, 1996. Detection of irradiated food containing bone method by ESR spectroscopy, EN 1786 (1996). Disponibile al sito: http://ec.europa.eu/ food/food/biosafety/irradiation/1786-1996_

Figura 2. Spettro relativo alle squame di barracuda non irradiate $(0 \mathrm{kGy})$ sperimentalmente irraggiate a $0,5 \mathrm{kGy}$. 


\section{en.pdf}

Commissione Europea, 1999a. Direttiva del Parlamento europeo e del Consiglio del 22 febbraio 1999 relativa al ravvicinamento delle legislazioni degli Stati membri concernenti gli alimenti e i loro ingredienti trattati con radiazioni ionizzanti, 1999/2/CE. In: Gazzetta Ufficiale, L 066, 13/03/1999.

Commissione Europea, 1999b. Direttiva del Parlamento europeo e del Consiglio del 22 febbraio 1999 che stabilisce un elenco comunitario di alimenti e loro ingredienti trattati con radiazioni ionizzanti, 1999/3/CE. In: Gazzetta Ufficiale, L 066, 13/03/1999.

Commissione Europea, 2000. Detection of irradiated food containing cellulose method by ESR spectroscopy, EN 1787 (2000). Disponibile al sito: http://ec.europa.eu/ food/food/biosafety/irradiation/17872000_en.pdf

Commissione Europea, 2001. Detection of irradiated food containing crystalline sugar by ESR spectroscopy, EN 13708 (2001).
Disponibile al sito: http://ec.europa.eu/ food/food/biosafety/irradiation/13708-2001 _en.pdf

Commissione Europea, 2012. The rapid alert system for food and feed. 2012 annual report. Commissione Europea ed., Lussemburgo.

Delincée H, 2002. Analytical methods to identify irradiated foods: a review. Radiat Phys Chem 63:455-8.

Eaton GR, Eaton SS, Salikhov KM, 1998. Foundations of modern EPR. World Scientific, Singapore.

European Food Safety Authority, 2011. EFSA panel on food contact materials, enzymes, flavourings and processing aids (CEF). EFSA J 9:1930.

FA0, 1984. Codex Alimentarius Commission. Codex general standard for irradiated foods and recommended international code of practice for the operation of radiation facilities used for the treatment of food. Vol. 15. Food and Agriculture Organization of the United Nations, Roma, Italia.

Farkas J, 1998. Irradiation as a method for decontaminating food: a review. Int J Food Microbiol 44:189-204.

Ikeya M, 1993. New applications of electron spin resonance. Dating, dosimetry and microscopy. World Scientific, Singapore.

Mangiacotti M, Marchesani G, Chiaravalle AE, 2013. Official checks by an accredited laboratory on irradiated foods at an Italian market. Food Control 33:307-12.

Repubblica Italiana, 2001. Decreto Legislativo 30 gennaio 2001, n. 94. Attuazione delle direttive 1999/2/CE e 1993/3/CE concernenti gli alimenti e i loro ingredienti trattati con radiazioni ionizzanti. In: Gazzetta Ufficiale n. 79, 4 aprile 2001.

Stefanova R, Vasilev VN, Sapassov SL, 2010. Irradiation of food, current legislation frame work and detection of irradiated foods. Food Anal Method 3:225-52.

WHO, 1999. High-dose irradiation: wholesomeness of food irradiated with dose above 10 kGy. World Health Organization, Ginevra, Svizzera. 